\section{LEVANTAMENTO ETNOZOOLOGICO DE VERTEBRADOS SILVESTRES TERRESTRES NA COMUNIDADE DO VAU DA BOA ESPERANÇA, BARREIRAS, OESTE DA BAHIA.}

\section{Kleiton Milton Lira ${ }^{1}$ Loyana Docio ${ }^{1} /$ Lourdes Marina Pessoa Bezerra ${ }^{1, *}$}

\section{RESUMO}

O conhecimento científico básico sobre grande parte da biodiversidade do Cerrado ainda é muito limitado, havendo grandes lacunas de informações fundamentais, como por exemplo a diversidade de espécie existente. Diante da intensa expansão do agronegócio na região Oeste da Bahia, muitas informações sobre a biodiversidade da região estão sendo perdidas. Assim, torna-se importante os estudos etnozoológicos como uma forma de colaborar no resgate destes dados. Nesse contexto, a presente pesquisa buscou resgatar informações históricas e atuais sobre a fauna silvestre do distrito do Vau da Boa Esperança, Barreiras, Bahia. O universo amostral contou com 15 entrevistados, todos eles leram e assinaram e termo de consentimento livre e esclarecido (TCLE). Durante as entrevistas foram citadas 52 espécies de animais diferentes, pertencentes a classe dos mamíferos, aves, répteis e anfíbios. Os animais mais citados durante as entrevistas foram as aves e os mamíferos, com 23 e 19 espécies, respectivamente. Também foram citados pelos entrevistados espécies que não são mais vistas na região, sendo os mamíferos mais citados, o que corrobora com estudos que aponta os mamíferos como sendo o que mais sofrem com a degradação do meio ambiente. Com o presente trabalho foi possível detectar uma grande variedade de espécies de animais silvestres que habitam ou já habitaram a região do Vau da Boa Esperança. Apesar de necessitar de mais investigações e detalhes sobre as informações obtidas pelos entrevistados, os resultados do presente estudo podem subsidiar futuros estudos faunísticos na região.

Palavras chaves: vertebrados silvestres, etnobiologia, cerrado, levantamento faunístico.

\section{ABSTRACT}

Basic scientific knowledge about much of the Cerrado's biodiversity is still very limited, with major information gaps such as the diversity of species present.
Given the intense expansion of agribusiness in the West region of Bahia, much information on the region's biodiversity is being lost. Thus, ethnozoological studies become important as a way to collaborate in the rescue of these data. In this context, the present research sought to recover historical and current information about the wildlife of the Vau da Boa Esperança district, Barreiras, Bahia. The sample universe had 15 interviewees, all of them read and signed and informed consent form. During the interviews 52 different species of animals belonging to the class of mammals, birds, reptiles and amphibians were mentioned. The most cited animals during the interviews were birds and mammals, with 23 and 19 species, respectively. Also cited by the interviewees were species that are no longer seen in the region, and mammals are most cited, which corroborates with studies that point to mammals as the most suffering from environmental degradation. With the present work it was possible to detect a great variety of species of wild animals that inhabit or already inhabited the Vau da Boa Esperança region. Despite the need for further investigation and details of the information obtained by the interviewees, the results of the present study may support future faunal studies in the region.

Keywords: wild vertebrates, ethnobiology, cerrado, faunistic survey.

Submetido em: 18 de jun. 2018

Aceito em: 06 de nov. 2018

\section{INTRODUÇÃO}

O Brasil é considerado como um dos países de maior diversidade biológica (Mittermeier et al., 1992). Em contraponto existe uma enorme carência de informações básicas sobre a distribuição desses recursos ao longo de suas diferentes regiões e biomas (Conservation International, 1993).

Os inventários e estudos faunísticos detalhados sobre vertebrados ainda são uma das fontes mais relevantes de dados de base para interpretações de padrões gerais de diversidade biológica (Lamoreux et al., 2006). Dados básicos sobre fauna são ainda mais urgentes em regiões pouco estudadas e sob intensa ameaça antrópica (Brooks et al., 2004), tais como a região de Cerrado, uma das 34 grandes regiões prioritárias para a conservação da biodiversidade global (Myers et al., 2000; Myers, 2003).

${ }^{1}$ Colegiado de Ciências Biológicas, Departamento de Ciências Humanas Campus IX, Universidade do Estado da Bahia, Barreiras, Bahia.

*E-mail para correspondência: lmarinabpessoa@gmail.com

Rev. ComCiência - dez. 2018, vol. 3, no. 3, p. 51-56/doi: 10.36112/issn2595-1890.v3i1.p51-56 
com Ciêncio

Uma revista multidisciplinar
Levantamento etnozoologico de vertebrados silvestres terrestres na comunidade do Vau da Boa Esperança, Barreiras, Oeste da Bahia
Na região Oeste da Bahia, o desenvolvimento do setor agrícola tem provocado profundas alterações na paisagem, principalmente no que se refere ao uso e ocupação da terra (Mendonça, 2006). Assim, muitas informações sobre a fauna nesta região vêm se perdendo de uma forma progressiva.

A etnozoologia é um ramo da etnobiologia que estuda os conhecimentos, significados e usos dos animais nas sociedades humanas (Overal, 1990). Para Rocha-Mendes et al. (2005) a etnozoologia é uma ferramenta interpretativa do histórico compartilhado entre o homem e os animais em uma determinada região. Neste sentindo, a etnozoologia se torna uma ferramenta importante para obter e resgatar informações relevantes sobre a fauna de uma determinada região.

A comunidade do Vau da Boa Esperança está inserida em uma região de floresta estacional decidual de transição entre a caatinga e o cerrado, no Oeste da Bahia. O mosaico da região também apresenta áreas de veredas e áreas de cerrado strictu sensu (Nascimento et al., 2015), tendo interferências humanas progressivas. Nas três últimas décadas, o Cerrado vem sendo degradado pela expansão da fronteira agrícola brasileira, notadamente na região Oeste da Bahia (Santos et al., 2009; Viana \& Bauch, 2009). Considerando a região do Vau da Boa Esperança uma área de cerrado característico e a intensa expansão do agronegócio na região Oeste da Bahia, os estudos etnozoológicos podem ser uma importante ferramenta no resgate dos dados sobre a fauna silvestre local, bem como para condução de estudos de levantamento e monitoramento de fauna em áreas remanescentes de Cerrado.
Nesse contexto, o trabalho teve como objetivo resgatar informações históricas e atuais sobre a fauna de vertebrados silvestres terrestres do distrito do Vau da Boa Esperança, Barreiras, Bahia.

\section{Material e Métodos}

A presente pesquisa foi realizada na comunidade do Vau da Boa Esperança, localizada no município de Barreiras, região oeste do estado da Bahia (12 $07^{\prime}$ 87-S $45^{\circ} 13^{\prime}$ 29-W), no período de agosto de 2016 a maio de 2017. A comunidade localiza-se a 37 $\mathrm{km}$ da sede do município, com mais de 150 anos de idade, a comunidade é composta por 24 moradores.

Os dados foram obtidos através de entrevistas abertas e semiestruturadas, com um total de 15 moradores residentes na comunidade entrevistados, sendo 11 homens e 4 mulheres, com idade entre 60 anos e acima de 65 anos (idosos). As entrevistas tiveram uma média de 40 minutos de duração, cada uma. Elas foram feitas seguindo os preceitos etnocientíficos com enfoque emiscista-eticista balanceado (Sturtevant, 1964), enquanto as observações foram um caráter ad libitum.

Todos os entrevistados ficaram cientes do Termo de Consentimento Livre Esclarecido (TCLE) concordando em fornecer as informações para o pesquisador, aprovado pelo Conselho de Ética da Universidade do Estado da Bahia (UNEB), sob o parecer $n^{\circ} 541.649$.

Os dados foram tabulados em planilhas do Microsoft Office Excel® e foi aplicado o Índice de Constância (Silveira-Neto et al. 1976) com intuito de verificar a popularidade das espécies nas entrevistas em forma de percentual. Dessa forma, as espécies pu- deram ser agrupadas em constantes (presentes em mais de $50 \%$ das entrevistas), acessórias (em 25 a 50\%) e ocasionais (em menos de $25 \%$ ).

\section{Resultados e Discussão}

Foram entrevistados 15 indivíduos, sendo 11 homens e 4 mulheres, com a maioria de idade até 60 anos e que residiam a mais de 10 anos na comunidade. Todos os entrevistados costumavam ver animais silvestres na área de estudo.

Durante as entrevistas foi possível listar uma grande variedade de espécies de animais citadas pelos entrevistados, com um total de 52 espécies de animais, pertencentes a classe dos mamíferos, aves, répteis e anfíbios. Tendo as aves e os mamíferos os maiores números de citações, com 23 e 19 espécies, respectivamente.

A classificação das espécies em ocasionais, acessórias ou constantes nas entrevistas, bem como os nomes pelos quais são localmente conhecidas, contam no Quadro 1. As espécies no qual não se conseguiu informações suficientes para classificação a nível específico foram apresentadas em nível genérico.

Diversos métodos podem ser utilizados para inventariar espécies de animais de uma determinada região, mesmo que estes não ocorram mais na área. Segundo Fonseca (2001), as entrevistas com moradores da área de estudo é considerado um método alternativo e de menor custo e tempo que os métodos tradicionais, como armadilhas e busca ativa, e que pode gerar informações atuais e passadas sobre determinadas espécies, além de nos fornecer informações etnozzológicas e biodinâmicas relacionadas a estes animais como podemos conferir 
Quadro 1: Lista, classes de ocorrência e status das espécies de mamíferos, aves, répteis e anfibios, ciom base em entrevistas realizadas no período de agosto de 2016 a maio de 2017 com 15 moradoreslocaisdo distrito do Vau da Boa Esperança, Barreiras, Bahia.

\begin{tabular}{|c|c|c|}
\hline Classe & Nomes populares locais & Ocorrência \\
\hline \multicolumn{3}{|l|}{ MAMÍFEROS } \\
\hline Mazama gouazoubira & veado & Constante \\
\hline Cerdocyon thous & raposa & Constante \\
\hline Puma concolor & onça & Constante \\
\hline Callithrix penicillata & mico & Constante \\
\hline Euphractus sexcintus & tatu-peba & Acessória \\
\hline Tayassu pecari & porco do mato & Acessória \\
\hline Coendou prehensilis & ouriço-cachero & Acessória \\
\hline Myrmecophaga tridactyla & tamanduá-bandeira & Acessória \\
\hline Conepatus semistriatus & gambá & Acessória \\
\hline Didelphis sp. & saruê & Acessória \\
\hline Alouatta caraya & guariba & Acessória \\
\hline Cuniculus paca & paca & Acessória \\
\hline Pecari tajacu & caititu & Ocasional \\
\hline Chrysocyon brachyurus & lobo-guará & Ocasional \\
\hline Kerodon rupestres & mocó & Ocasional \\
\hline Procyon lotor & guaxinim & Ocasional \\
\hline Nasua nasua & quati & Ocasional \\
\hline Leopardus pardalis & jaguatirica & Ocasional \\
\hline Cavia sp. & preá & Ocasional \\
\hline \multicolumn{3}{|l|}{ AVES } \\
\hline Rhea amaricana & ema & Constante \\
\hline Amazona sp. & papagaio & Constante \\
\hline Penelope ochrogaster & јасu & Constante \\
\hline Ara sp. & arara & Constante \\
\hline Eupsitulla aurea & periquito & Constante \\
\hline Cariama cristata & seriema & Acessória \\
\hline Nothura boraquira & codorna & Acessória \\
\hline Leptotila verreauxi & juriti & Acessória \\
\hline Ramphastos toco & tucano & Acessória \\
\hline Turdus rufiventris & sabiá & Acessória \\
\hline Tyto alba & coruja & Acessória \\
\hline Cyanocorax cyanopogon & cancão & Ocasional \\
\hline Columbina picui & rolinha & Ocasional \\
\hline Gnorimopsar chopi & graúna & Ocasional \\
\hline Crypturellus parvirostris & nambu & Ocasional \\
\hline Patagioenas picazuro & pomba & Ocasional \\
\hline Caracara plancus & gavião & Ocasional \\
\hline Herpetotheres cachinnans & coã & Ocasional \\
\hline Sicalis flaveola & canário & Ocasional \\
\hline Zonotrichia capensis & tico-tico & Ocasional \\
\hline Paroaria coronata & cardeal & Ocasional \\
\hline Crypturellus noctivagus & zabelê & Ocasional \\
\hline Columbina squammata & fogo-apagou & Ocasional \\
\hline \multicolumn{3}{|c|}{ RÉPTEIS E ANFÍBIOS } \\
\hline Crotalus sp. & cascavel & Constante \\
\hline Brothrops sp. & jararaca & Constante \\
\hline Tropidurus sp. & lagartixa & Constante \\
\hline Bufo sp. & sapo & Constante \\
\hline Ameivula sp. & Calango & Constante \\
\hline Tupinambis merianae & Teiú & Constante \\
\hline Leptodactylus sp. & rã & Constante \\
\hline Iguana iguana & iguana & Constante \\
\hline Scinax sp. & perereca & Acessória \\
\hline Caiman sp. & jacaré & Acessória \\
\hline
\end{tabular}

nos estudos de Vidolin et al., 2002, Conforti \& Azevedo, 2003, Pedroso Jr. \& Sato 2003, Rocha-Mendes et al., 2005, Oliveira \& Calixto Jr., 2016).

Quando questionados sobre os animais que mais encontravam próximos as suas residências, foram mencionadas 14 espécies de animais diferentes, contemplando as classes de mamíferos, aves, anfíbios e répteis. Das espécies mais mencionadas nas entrevistas, destaca-se as cobras, seguida dos veados e dos micos.

De acordo Ellen (1997), o conhecimento zoológico tradicional pode variar qualitativa e quantitativamente, inclusive de acordo com o gênero, faixa etária e nível de empatia com o animal. Isso pode justificar a grande quantidade de espécies de aves e mamíferos citadas durante as entrevistas, uma vez que, as aves e os mamíferos são caracterizadas como animais carismáticos e de grande empatia pelos humanos. Em contrapartida, os répteis e os anfíbios são percebidos como feios, nojentos e potencialmente capazes de transmitir doenças (Souza e Souza, 2006).

Segundo os entrevistados, muitos animais foram desaparecendo com o tempo, principalmente nos últimos 10 anos, o que coincide com a expansão agropecuária na região. Segundo um documento oficial do governo brasileiro, as atividades agropecuárias, junto com a siderurgia, já levaram à perda de aproximadamente metade da área original de vegetação nativa do Cerrado (MMA, 2009), o que acarreta em uma perda gigantesca de biodiversidade devido a fragmentação do Cerrado e a substituição de área nativa por monoculturas e pastagens.

Dentre os animais citados pelos entrevistados que desapareceram da região estudada ou foram vistos 
com Ciêncio

Uma revista multidisciplinar
Levantamento etnozoologico de vertebrados silvestres terrestres na comunidade do Vau da Boa Esperança, Barreiras, Oeste da Bahia muito pouco nestes últimos 10 anos, estão o veado galheiro (Blastocerus dichotomus) (26,66\%), o cacheiro (Coendou prehensilis) $(26,66 \%)$ e o tamanduá bandeira (Mymercophaga tridactyla ) (20\%), além da Ema (Rhea americana) $(13,33 \%)$, do porco do mato (Pecari tajacu) $(13,33 \%)$ e do tatu canastra (Priodeontes maximus) $(13,33 \%)$. Observa-se com esses dados uma grande variedade de mamíferos vem desaparecendo nestes últimos 10 anos, o que pode estar relacionado a velocidade de desmatamento e fragmentação devido a ação antrópica no Cerrado, como citou Machado et al. (2004). Sendo, assim, os mamíferos de médio e grande porte $(\geq 1 \mathrm{~kg}$ segundo Fonseca \& Robinson, 1990) são afetados pela fragmentação e alteração do habitat decorrente da ocupação humana (Rodrigues et al. 2002, Trolle et al. 2007) que, juntamente com a pressão de caça, correspondem às principais ameaças a esse grupo (Costa et al. 2005). Quando analisamos o nível de ameaças destes mamíferos citados, observamos que apenas o cacheiro (Coendou prehensilis) não se encontra em nível de alerta de ameaça de extinção (MMA, 2008). O que podemos relacionar com o nível de destruição de habitat e pelo desmatamento da região, corroborando com os dados do MMA (2009) onde indica que a maioria das espécies de mamíferos estão ameaçadas de extinção principalmente pela destruição de habitat e desmatamento, sendo mais intenso nos biomas Cerrado e Mata Atlântica.

A fragmentação de habitat refere-se às mudanças na configuração do ambiente resultantes de uma quebra de continuidade da passagem, independente da perda ou não de habitat (Fahrig, 2003). Esse processo pode trazer efeitos importantes sobre as espécies da fauna silvestres, dentre eles podemos citar a redução e/ou isolamento de área, o aumento do efeito de borda e a redução do número de ambientes que afetam diretamente no tamanho da população, reduzindo a viabilidade genética e podem provocar distúrbios para os níveis tróficos (Terborgh, 1992; Fahrig, 2003) e mesmo desaparecimentos de espécies.

Resultados dessa fragmentação na área do estudo foi demonstrada pelos entrevistados quando perguntados sobre os animais que não são mais vistos na região, sendo os mais citados os mamíferos como o veado galheiro (Blastocerus dichotomus), o tamanduá bandeira (Mymercophaga tridactyla) $e$ $o$ tatu canastra (Priodeontes maximus), que são animais que possuem distribuição pela região. As respostas dos participantes da pesquisa foram congruentes às análises sobre perda da diversidade biológica, como consta no livro vermelho da fauna silvestre ameaçada de extinção (MMA, 2008). Assim, com o estudo foi possível obter dados sobre alterações temporais na composição da fauna silvestre local, sugerindo uma extinção local dessas espécies.

O desaparecimento de espécies grandes, sobretudo de mamíferos está diretamente relacionada com a disponibilidade de área de forrageamento (Tabarelli \& Gascon, 2005). Assim, com o aumento das áreas agricultáveis no oeste da Bahia, estes organismos perderam espaço, de forma a ser percebido suas ausências pela população local. Observa-se que entre os anos de 2002 a 2009, cerca de 6.260 $\mathrm{km}^{2}$ do cerrado baiano foi desmatado. Isso corresponde a cerca de $30 \%$ de sua dimensão na Bahia (Silva et al., 2011).

\section{Conclusão}

As abordagens etnozoológicas utilizadas no presente trabalho foram eficientes para obtenção das informações históricas e atuais sobre a fauna silvestre de vertebrados terrestres do distrito do Vau da Boa Esperança. Podendo, assim, ser utilizado como ferramenta para estudos de inventários na região.

Com o presente trabalho foi possível detectar uma grande variedade de espécies de animais silvestres que habitam ou já habitaram a região do Vau da Boa Esperança, Barreiras, Bahia. Os dados obtidos quanto às extinções locais de algumas espécies e a alteração na composição faunística local, demonstra a eficiência da etnozoologia no diagnóstico de problemas relacionados a conservação da fauna. Além de inferir que a fragmentação do Cerrado, com o aumento das áreas desmatadas, influenciou no desaparecimento destas espécies.

Embora exista a necessidade de estudos mais detalhados e investigação de algumas informações obtida pelos entrevistados, os resultados obtidos nesta pesquisa podem subsidiar futuros estudos etnozoológicos e faunísticos na região.

\section{Agradecimentos}

À Universidade do Estado da Bahia (UNEB), ao programa de iniciação pela oportunidade e bolsa concedida PICIN, a orientadora, aos amigos que me acompanharam nas pesquisas de campo e aos moradores da Comunidade estudada. 
com Ciência

Uma revista multidisciplinar
Levantamento etnozoologico de vertebrados silvestres terrestres na comunidade do Vau da Boa Esperança, Barreiras, Oeste da Bahia

\section{Referências}

BROOKS, T.; FONSECA, G.; RO-

DRIGUES, A. Species, data, and conservation planning. Conserv. Biol. 18: 1682-1688, 2004.

\section{CONFORTI, V.A. \& AZEVEDO,}

F.C.C. Local perceptions of jaguars (Panthera onca) in the Iguaçu National Park area, south Brazil. Biological Conservation, Essex, 2003, 111 (2): 215-221.

\section{CONSERVATION INTERNATIO-} NAL, FUNDAÇÃO BIODIVERSIDADE \& SOCIEDADE NORDESTINA DE ECOLOGIA. Prioridades para a conservação da biodiversidade da Mata Atlântica do Nordeste. Mapa descritivo produzido pela CI, FB e SNE. 1993.

COSTA, L.P., LEITE, Y.L.R., MENDES, S.L. \& DITCHFIELD, A.B. Conservação de mamíferos no Brasil. Megadiversidade 1(1):103-112. 2005.

FAHRIG, L. Effects of habitat fragmentation on biodiversity. Annual Review of Ecology, Evolution and Systematics, 2003, 34: 487-515.

FONSECA, G.A.B. \& Robinson, J.G. 1990. Forest size and structure: competitive and predatory effects on small mammal communities. Biol. Conserv. 53:265-294.

FONSECA, G.A.B. Proposta para um programa de Avaliação Rápida em âmbito nacional. In: GRAY, I. \& DIAS, B. (Eds). Conservação da biodiversidade em ecossistemas tropicais, Petrópolis, Editora Vozes, 2001.
LAMOREUX, J.; MORRISON, J.; RICKETTS, T.; OLSON, D.; DINERSTEIN, E.; MCKNIGHT, M.; SHUGART, H. Global tests of biodiversity concordance and the importance of endemism. Nature 440:212-214. 2006.

MACHADO, R. B.; RAMOS NETO, M. B.; PEREIRA, P. G. P.; CALDAS, E.F.; GONÇALVES, D.A.; SANTOS, N.S.; TABOR, K. \& STEININGER,

M. Estimativas de perda da área do Cerrado brasileiro. Conservação Internacional do Brasil, Brasília. Relatório técnico, 2004.

MENDONÇA, J. O. O potencial do crescimento da produção de grãos no oeste da Bahia. Bahia Agrícola, v. 7, n. 2, p. 38-46, 2006.

\section{MITTERMEIER, R.; AYRES, J.;} WERNER, F. O País da Megadiversidade. Ciência Hoje, 14:20-27.1992.

MMA (Ministério do Meio Ambiente). Livro vermelho da fauna brasileira ameaçada de extinção / editores Angelo Barbosa Monteiro Machado, Gláucia Moreira Drummond, Adriano Pereira Paglia. - 1.ed. - Brasília, DF : MMA; Belo Horizonte, MG : Fundação Biodiversitas, 2008.

MMA (Ministério do Meio Ambiente). Relatório Técnico de Monitoramento de desmatamento do bioma Cerrado, 2002 a 2008: Dados Revisados. Ministério do Meio Ambiente, Brasília. 2009.

MYERS, Norman.Biodiversity hotspots revisited. Bioscience 53:916917. 2003.

MYERS, Norman; MITTERMEIER,
R.A., MITTERMEIER, C.G., FONSECA G.A.B. \& KENTS, J. Biodiversity hotspots for conservation priorities. Nature 403:853-858. 2000.

NASCIMENTO, V.; PEREIRA, H.; SILVA, A.; NUNES, A.; MEDEIROS, P. Plantas alimentícias espontâneas conhecidas pelos moradores do Vau da Boa Esperança, município de Barreiras, oeste da Bahia, nordeste do Brasil. Revista Ouricuri. V.5, n.1, 2015.

OLIVEIRA, J. O. \& CARLIXTO JR., J.T. Levantamento etnozoológico junto à população do município de Araripe, Sul do Ceará. Caderno de Cultura e Ciência, 15 (2), 2016.

OVERAL, W. L. Introduction to ethnozoology: what it is or could be. In: Posey, D. A. \& Overal, W. L. (orgs.). Ethnobiology: implications and applications. MPEG, Belém, Brasil, 1990. p.127-129.

PEDROSO JR., N. N. \& SATO, M. Percepção de fauna terrestre e conservação no Parque Nacional do Superagui. Revista de Educação Pública, Cuiabá, 2003, 12 (21): 43-70.

ROCHA-MENDES, F.; MIKICK, S. B.; BIANCONI, G. V. \& PEDRO, W.A. Mamíferos do município de Fênix, Paraná, Brasil: etnozoologia e Conservação. Revista brasileira de Zoologia 22 (4): 2005.

RODRIGUES, F.H.G., SILVEIRA, L., JÁCOMO, A.T.A., CARMIGNOTTO, A.P., BEZERRA, A.M.R., COELHO, D.C., GARBOGINI, H., PAGNOZZI, J. \& HASS, A. Composição e caracterização da fauna de mamíferos do Parque Nacional das Emas, Goiás, Brasil. Rev. Bras. Zool. 19(2):589- 
com Ciêncio

Uma revista multidisciplinar
Levantamento etnozoologico de vertebrados silvestres terrestres na comunidade do Vau da Boa Esperança, Barreiras, Oeste da Bahia
600. 2002

SANTOS, P.M.C, ALVES M.S., SILVA D.A., CARNEIRO, C.L. \& FERNANDES, M. M. Monitoramento do desmatamento no Cerrado, porção oeste da Bacia do Rio São Francisco: uma análise dos produtos NDVI e Modelo de Mistura Espectral. In: XIV Simpósio Brasileiro de Sensoriamento Remoto, Natal, RN. Anais... São José dos Campos, SP, INPE. 2009.

SILVA, E. B; FERREIRA, L. G.; ROCHA, G. F.; ANJOS, A. F. O desmatamento do Extremo Oeste Baiano e sua correlação com a produção de grãos entre 2005 e 2007 vistos a partir de dados orbitais e dados censitários. Anais do simpósio Brasileiro de sensoriamento remoto. 6349-6357. Curitiba, 2011.

SILVEIRA-NETO, S.; NAKANO, O.; BARBIN, D. \& NOVA, N.A.V. Manual de ecologia dos insetos. São Paulo, Editora Agronômica. 1976.

SOUZA, C. E. P.; SOUZA, J. G. (Re) conhecendo os animais peçonhentos: diferentes abordagens para a compreensão da dimensão histórica, sócioambiental e cultural das ciências da natureza. 2006.

STURTEVANT, W. Studies in Ethnoscience. American Anthropologist, v.66, n. 3, p. 99-131, 1964.

TABARELLI, M.; GASCON, C. Lições da pesquisa sobre fragmentação: aperfeiçoando políticas e diretrizes de manejo para a conservação da biodiversidade. Megadiversidade 2005; 1(1): 181-188.
TERBORGH, J. Maintenance of diversity in tropical forest. Biotropica, 24 (2b): 283-292. 1992.

TROLLE, M., BISSARO, M.C. \& PRADO, H.C. Mammal survey at a ranch of the Brazilian Cerrado. Biodivers. Conserv. 16(4):1205-1211. 2007.

VIANA, S.N. \& BAUCH, A. A. O uso de imagens CBERS no monitoramento da cobertura vegetal da bacia hidrográfica do rio São Francisco - estudo de caso: Oeste Baiano. In: XIV Simpósio Brasileiro de Sensoriamento Remoto, Natal, RN. Anais. São José dos Campos, SP, INPE. 2. Municípios localizados no Bioma Cerrado e a área desmatada no ano de 2009.

VIDOLIN, G.P.; UCHÔA, P.R.; MANGINI, F.R.; MENDES, A.M.; KUCZACH \& BORLACHENCO,

N.G.C. Felinos na Reserva Natural Salto Morato, Guaraqueçaba, Paraná. Brasil: Levantamento e caracterização de seus aspectos ecológicos. In: M.S. MILANO \& V. THEULEN (Eds.). Anais II Congresso Brasileiro de Unidades de Conservação. Campo

GrANDE, 2002. 